\title{
Exceptional toxicity resistance
}

\author{
Stephen Sonis ${ }^{1}$ - Alessandro Villa ${ }^{2}$ - Joel Epstein ${ }^{3,4}$
}

Published online: 16 February 2021

(C) The Author(s), under exclusive licence to Springer-Verlag GmbH, DE part of Springer Nature 2021

Over ninety percent of patients being treated with chemoradiation for cancers of the head and neck develop ulcerative mucositis, and more than $65 \%$ suffer from mucositis of such severity that alimentation of solid foods, even soft solids, is impossible $[1,2]$. Oral mucositis is representative of many of the regimenrelated toxicities of which we have studied and described the demographic, epidemiologic, proteomic, microbiomic, and genomic features of patients afflicted with these conditions in search of factors influencing risk, pathobiology, and outcomes. Yet, we have largely ignored that small percentage of patients who, despite having the same cancer diagnosis, the same treatment, and the same demographics, never manifest measurable subjective or objective evidence of the toxicity of interest. In the case of mucositis, these patients retain pink and intact mucosa, have no diet limitations, maintain their weight, and sail through treatment symptom-free. What underlies the reasons that result in this small, but recognizable cohort? And while mucositis is an established example of a clinically significant regimen-related toxicity, the finding of a small cohort of patients who withstand even the most aggressive cytotoxic regimens without any manifestation of adverse outcomes is wellknown. It seems likely that this select group of exceptional responders might be a source of data which differentiates toxicity risk and provides information around toxicity clustering and pathogenesis that could also provide a conduit for insights on effective therapeutic options.

The concept of identifying exceptional responders in patients being treated for cancer and using molecular and bio-

Stephen Sonis

ssonis@biomodels.com

1 Divisions of Oral Medicine and Dentistry, Brigham and Women's Hospital and the Dana-Farber Cancer Institute, Boston, MA, USA

2 Department of Orofacial Sciences, University of California at San Francisco, San Francisco, CA, USA

3 City of Hope Comprehensive Center, Duarte, CA, USA

4 Cedars-Sinai Medical System, Los Angeles, CA, USA logical outcomes which characterize responders is not new. In 2014, the National Cancer Institute (NCI) launched the Exceptional Responders Initiative which evolved from a pilot program in which Phase 2 results identified 100 patients who were classified as exceptional responders to their therapy. Subsequently, the NCI established specific criteria to define exceptional responders based on having met at least one of the following criteria [3] for patients being treated for any type of cancer [4]:

1. $10 \%$ or fewer patients receiving the specific regimen had a complete or durable partial response lasting at least 6 months.

2. Patients achieved either a complete or partial response lasting at least 6 months as defined by RECIST (Response Evaluation Criteria in Solid Tumors) or similar criteria.

3. Patients had a sustained complete or partial response 3 times longer than the median duration of response based on comparative data from the literature.

As the NCI initiative continues to identify and enroll patients, Nishikawa et al. performed a comprehensive literature review to describe the frequency and characteristics of exceptional responders and found 180 cases, mostly patients diagnosed with breast cancer, that were identifiable [5].

Using tissue from an unbiased cohort identified through the NCI initiative, Wheeler et al. have recently reported the molecular mechanisms potentially associated with exceptional response to therapy using genetic and epigenetic tumor profiling [6]. Their report delineated the potential that data from this unique population can be used to define mechanism and identify potential therapeutic targets and identified while redundant pathways can circumvent treatment effect in most of the population; such was not the case in the exceptional responder group.

Could such an approach be used in the context of regimenrelated toxicities? It would seem so. The first challenge would be agreement on the definition of an "exceptional responder." 
While the NCI focused on tumor diagnosis, a supportive care approach would be driven by the expected response to a particular treatment regimen. Thus, we might define a mucositis exceptional responder as a patient being treated with a standard concomitant chemoradiation regimen for oropharyngeal cancer who never develops ulceration (WHO score $<2$ ) throughout the treatment period and for up to 4 weeks thereafter (about $6 \%$ of patients). Furthermore, rather than settle on a single toxicity, we might want to consider a toxicity cluster (e.g., for the CRT-HNC patient-no mucositis or dermatitis).

A second task would be to determine the variables that might be associated with exceptional toxicity resistance. These would certainly be associated with patient-centric variables like genomics, but might also include microbiomics, demographics, comorbidities, concomitant medications, diet, and socioeconomic factors. Tumor-based biological contributions to systemic responses to therapy are virtually unexplored, but given the potential activity attributable to tumors, it seems naïve to ignore their characterization as toxicity facilitators or catalysts.

Given the collective number of cancer treatment regimens and the number of potential toxicities ( $n=838$ individual toxicities are described by NCI-CTCAE v.5), creation of a hierarchical matrix of targeted individual and clustered toxicities would be critical, along with specific clinical, laboratory, and tissue information that would need to be collected. Since this information is probably most informative in treatment patients, a prospective approach would be necessary. Not a small undertaking as, using the CRT-HNC example, one would need to collect data and samples from 100 patients to yield 6 evaluable sets. This yield would become even more constricted in the assessment of toxicity cluster resistance. Nonetheless, as has been argued by many, prospective data and sample collection as a matter of practice has the potential of enormous benefits and has largely driven the creation of tumor banks at many institutions. For the purposes described here, one would require, in addition to tumor samples, patients' germline RNA, samples for microbiome characterization, serum for biomarker analysis, and organized, complete, and standardized clinical information. Importantly, consistent toxicity assessment and documentation would be critical to assure uniformity of the exceptional responder population.

While we should not abandon our studies of toxicity-laden patients, there is little doubt that the study of exceptional responders could produce information which could define interventional targets, better define the systems medicine relationships that impact toxicities, and provide mechanistic material which could impact not only cancer patients but also pathologies with common pathobiology and phenotypes.

\section{Declarations}

Conflict of interest Dr. Sonis is an employee of Biomodels, LLC and Primary Endpoint Solutions, LLC. Both companies assist industry, government, and academics to study and enable drugs, biologicals, and devices to treat patients for a broad range of indications including toxicities of cancer therapy. Dr. Sonis does not have equity or receive payment from any clients.

Dr. Villa serves as a clinical indication expert for Primary Endpoint Solutions, LLC.

Dr. Epstein serves on an advisory role for Galera Therapeutics, Tosk Pharma, and Rakuten Medical.

\section{References}

1. Sroussi HY, Epstein JB, Bensadoun RJ, Saunders DP, Lalla RV, Migliorati CA, Heaivilin N, Zumsteg ZS (2017) Common oral complications of head and neck cancer radiation therapy: mucositis, infections, saliva change, fibrosis, sensory dysfunctions, dental caries, periodontal disease, and osteoradionecrosis. Cancer Med 6:29182931

2. Villa A, Sonis S (2016) Toxicities associated with head and neck cancer treatment and oncology-related clinical trials. Curr Probl Cancer 40:244-257

3. Tsimberidou AM, Said R, Staudt LM, Conley BA, Takebe N (2019) Defining, identifying, and understanding "exceptional responders" in oncology using the tools of precision medicine. Cancer J 25:296-299

4. Conley BA, Staudt L, Takebe N, Wheeler DA, Wang L, Cardenas MF, Korchina V, Zenklusen JC, McShane LM, Tricoli JV, Williams PM, Lubensky I, O’Sullivan-Coyne G, Kohn E, Little RF, White J, Malik S, Harris LN, Mann B, Weil C, Tarnuzzer R, Karlovich C, Rodgers B, Shankar L, Jacobs PM, Nolan T, Berryman SM, GastierFoster J, Bowen J, Leraas K, Shen H, Laird PW, Esteller M, Miller V, Johnson A, Edmondson EF, Giordano TJ, Kim B, Ivy SP (2021) The exceptional responders initiative: feasibility of a national cancer institute pilot study. J Natl Cancer Inst 113:27-37

5. Nishikawa G, Luo J, Prasad V (2018) A comprehensive review of exceptional responders to anticancer drugs in the biomedical literature. Eur J Cancer 101:143-151

6. Wheeler DA, Takebe N, Hinoue T, Hoadley KA, Cardenas MF, Hamilton AM, Laird PW, Wang L, Johnson A, Dewal N, Miller V, Piñeyro D, Castro de Moura M, Esteller M, Shen H, Zenklusen JC, Tarnuzzer R, McShane LM, Tricoli JV, Williams PM, Lubensky I, O'Sullivan-Coyne G, Kohn EC, Little RF, White J, Malik S, Harris L, Weil C, Chen AP, Karlovich C, Rodgers B, Shankar L, Jacobs P, Nolan T, Hu J, Muzny DM, Doddapaneni H, Korchina V, GastierFoster J, Bowen J, Leraas K, Edmondson EF, Doroshow JH, Conley BA, Ivy SP, Staudt LM (2021) Molecular features of cancers exhibiting exceptional responses to treatment. Cancer Cell 39:3853 e7

Publisher's note Springer Nature remains neutral with regard to jurisdictional claims in published maps and institutional affiliations. 